\title{
Phase Retrieval Imaging for Soft Materials at Low-Voltage
}

Kelly Parker ${ }^{1}$, Blaise Kimmel ${ }^{2}$, Milan Mrksich ${ }^{3}$, Roberto dos Reis ${ }^{4}$ and Vinayak Dravid ${ }^{4}$

${ }^{1}$ Department of Materials Science and Engineering, Northwestern University, Evanston, Illinois, United States, ${ }^{2}$ Department of Chemical and Biological Engineering, Northwestern University, Chicago, Illinois, United States, ${ }^{3}$ Department of Biomedical Engineering, Northwestern University, United States, ${ }^{4}$ Department of Materials Science and Engineering, Northwestern University, United States

The imaging of soft and biological materials has long challenged researchers due to their low inherent contrast and susceptibility to beam damage even at low electron dose. Cryo-transmission electron microscopy (TEM) and advances in related hardware, software and sample preparation methods have greatly improved resolution and signal-to-noise for the imaging of soft structures. However, achieving sufficient contrast is generally dependent on lens aberrations or the introduction of phase plates to induce phase contrast [1], or on algorithms for class averaging from highly homogeneous samples. Scanning transmission electron microscopy (STEM) can be used to observe mass-thickness contrast, but the high localized dose can be prohibitive for radiation-limited structures. Imaging at lower voltage can improve contrast due to the increased scattering cross-section but also introduces elevated radiation damage [2]. The imaging of materials that are non-homogeneous or flexible in solution as well as beam-sensitive requires extended methods to improve contrast without increasing electron dose. One such method is ptychography, a phase retrieval imaging technique popularized for scanning x-ray diffraction microscopy [3]. It has recently proven useful in electron microscopy, especially for low-atomic number, easily damaged materials.

This work focuses on a class of materials known as "megamolecules", which are protein assemblies formed from covalent reactions between small molecule inhibitors and their target protein domains [4]. The model system used here, tetracutinase, is formed from the reaction of four cutinase protein domains and a four-armed poly(ethylene glycol) linker that is terminated with cutinase inhibitors (shown in Figure 1a), which yields a distinctive four-lobed architecture. Our previous work has shown images of tetracutinase using STEM-in-SEM at voltages from 5-30 kV [5]. A ptychography experimental setup is shown schematically in Figure 1 for a simulated image of tetracutinase on a graphene oxide thin film. Farfield electron diffraction patterns are recorded for each probe position, and a phase-contrast image of the structure can be reconstructed using the exit plane wave function. Changing the convergence angle prioritizes high or low spatial frequencies, and images according to different scattering angles can be reconstructed as analogues to various STEM modes [6]. Similarly to conventional TEM and STEM imaging, either a focused (as in Figure 1a) or defocused probe can be used for ptychographic reconstruction [1]. Using a focused probe allows for multimodal imaging in conjunction with STEM, while a defocused probe can be applied to further reduce electron dose. Phase retrieval imaging has been performed on a number of inorganic materials, including defocused ptychography of two-dimensional structures [7]; recent studies have also demonstrated the promise of ptychography for biological structures such as rotavirus [6] and various macromolecules [8] at lower dose than in conventional TEM or STEM.

Tetracutinase is used to exemplify simulated ptychography reconstructions at low voltages, including those achieved in a typical SEM setup (e.g. 5-30kV), and this technique can be applied to improve the visualization of more complex structures. Figure 2 shows simulated phase retrieval images at $5 \mathrm{kV}$ and 20 $\mathrm{kV}$ for doses on the order of 1 and $10^{3} \mathrm{e}^{-} / \AA^{2}$. The ptychographic simulations employed the single-side band (SSB) method [9] with aberration free data and a $30 \mathrm{mrad}$ aperture semi-angle. These phase images 
are compared to dark field (DF) and annular bright field (ABF) images at the same electron doses. Results highlight the benefit of extremely low-voltage imaging. While the full object can be retrieved at doses similar to traditional imaging $\left(10^{3} \mathrm{e}^{-} / \AA^{2}\right)$, biological materials are easily damaged at this dose; for homogeneous structures, class averaging can be used to enhance contrast and single particle analysis can be performed using a much smaller number of images and distinct particles than conventionally in cryoTEM [8]. Improving contrast can be achieved by exploring different reconstruction algorithms, convergence angles, and probe sizes. Phase reconstruction offers higher contrast at lower dose than DF or $\mathrm{ABF}$ for both $5 \mathrm{kV}$ and $20 \mathrm{kV}$ and is promising for non-homogeneous or flexible structures where class averaging is not possible. One of the primary barriers for low-voltage imaging, particularly below 5-10 $\mathrm{kV}$ using STEM-in-SEM, is the prevalence of chromatic aberration; ptychography reconstruction also allows for chromatic aberration correction [10].

This work demonstrates the promise of phase retrieval imaging for macromolecular complexes at low voltage, particularly in SEM. While this method was shown for a model system that has already been characterized using STEM-in-SEM, it can be applied to a variety of complex structures whose flexibility or inhomogeneity limit imaging using cryo-TEM. Ptychography can also be combined with spectroscopy to simultaneously analyze materials from both a morphological and chemical perspective. It is additionally promising in capturing localized processes that cannot be averaged for traditional contrast enhancement, such as an enzymatic reaction inducing a conformational change. The efficacy of ptychography in characterizing soft materials is only just being explored, and further work will critically examine the effect of voltage, electron dose, and a variety of other factors on the experimental ptychographic reconstruction of complex soft and biological structures

Acknowledgments: This work is supported by the Army Research Office (W911NF1810200), the National Science Foundation Graduate Research Fellowship (DGE-1842165), and the Ryan Fellowship, and partially supported by the Air Force Office of Scientific Research (FA9550-12-1-0280).
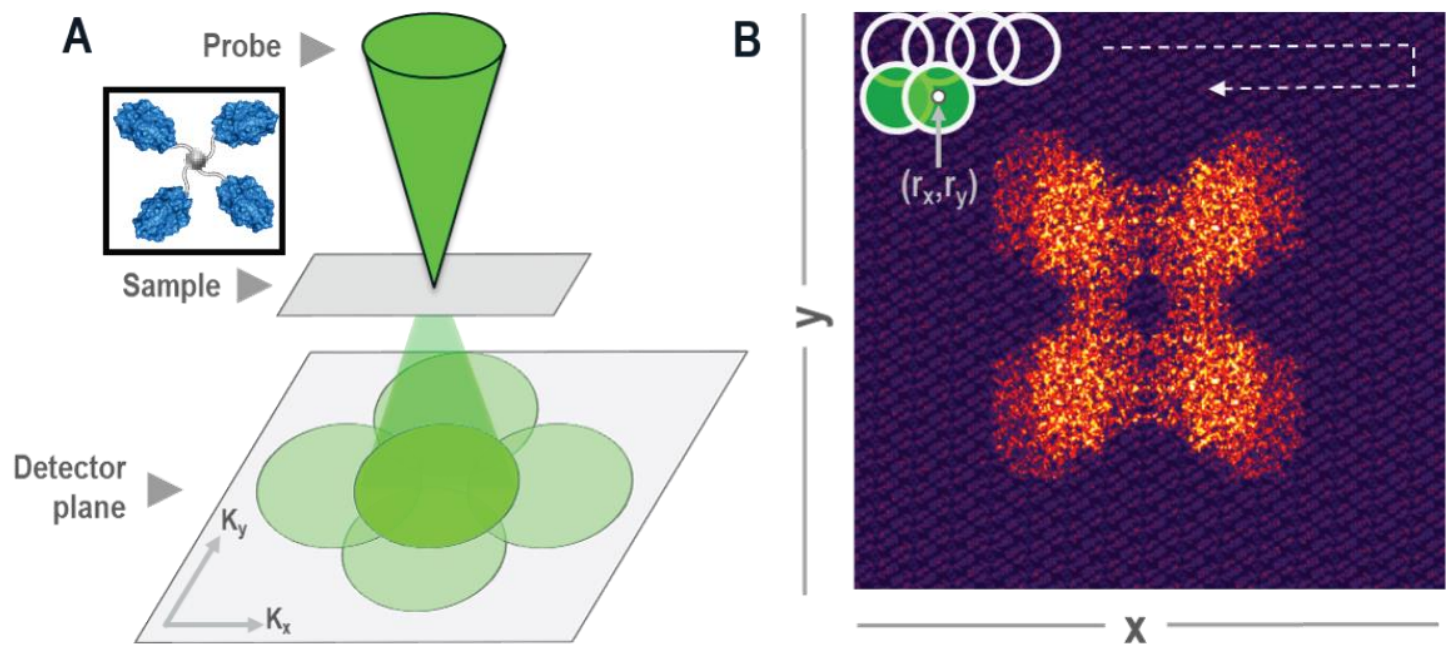

Figure 1. A) Schematic of ptychographic setup using a focused probe, where far-field diffraction patterns are collected at the detector plane for each probe position. Inset shows schematic of tetracutinase. B) Simulated image of tetracutinase on a graphene oxide thin film showing example diffraction collection points. Full image width is $17.1 \mathrm{~nm}$. 


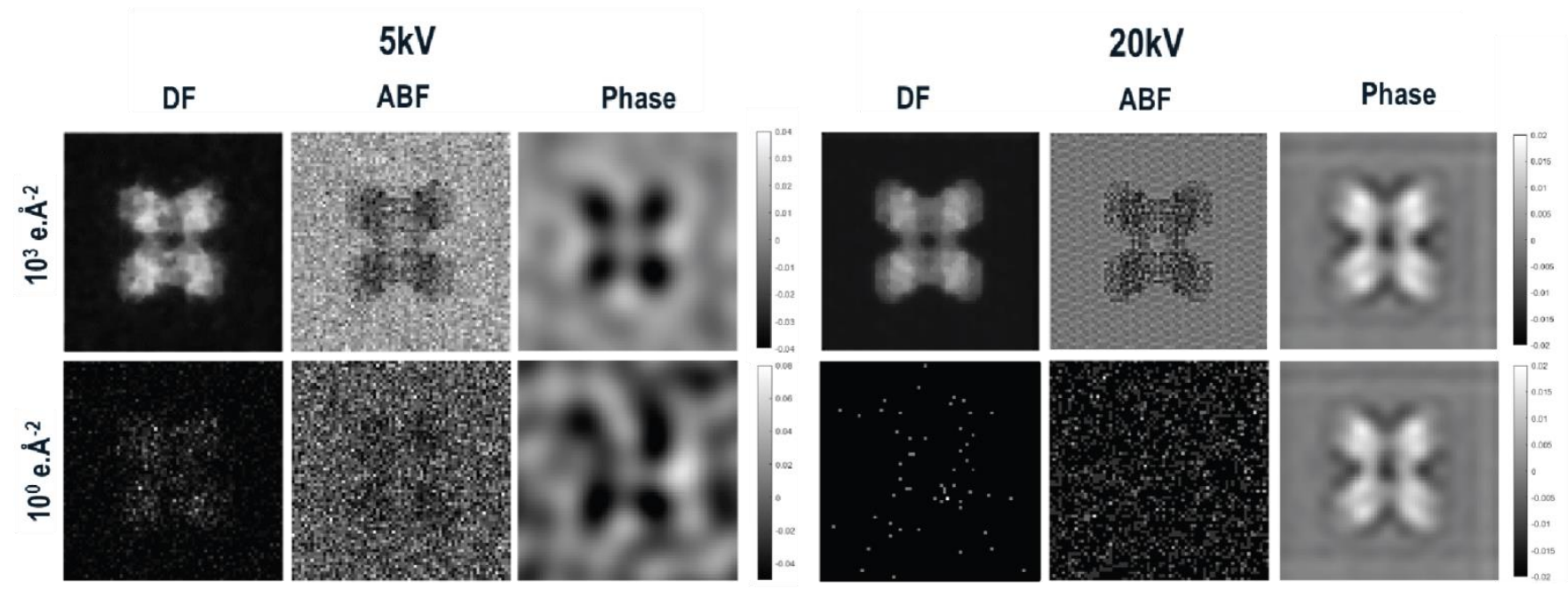

Figure 2. Simulated dark field, annular bright field and phase ptychographic reconstructions of tetracutinase on graphene oxide for $5 \mathrm{kV}$ and $20 \mathrm{kV}$ at doses on the order of 103 and $100 \mathrm{e}-/ \mathrm{A} 2$. DF and $\mathrm{ABF}$ images are normalized, and phase image scale is in units of rads. Full image width is $17.1 \mathrm{~nm}$.

References

[1] Pennycook T. J., Martinez G. T., Nellist P. D., Meyer J. C. Ultramicroscopy 196 (2019), p. 131-135.

[2] Bell D. C., Mankin M., Day R. W., Erdman N. Ultramicroscopy 145 (2014), p. 56-65.

[3] Thibault P., Dierolf M., Menzel A., Bunk O., David C., Pfeiffer F. Science 321, 5887 (2008), p. 379382.

[4] Modica J. A., Skarpathiotis S., Mrksich M. ChemBioChem 13, 16 (2012), p. 2331-2334.

[5] Parker K., Zhou S., Mrksich M., Dravid V. et al. Microscopy and Microanalysis 26, S2 (2020), p. 1026-1028.

[6] Zhou L., Song J., Wang P. et al. Nature Communications 11, 2773 (2020).

[7] Song J., Allen C. S., Kirkland A. et al. Scientific Reports 9, 3919 (2019).

[8] Pelz P. M., Qiu W. X., Bucker R., Gunther K., Miller R. J. D. Scientific Reports 7, 9883 (2017).

[9] Pennycook T. J., Lupini A. R., Nellist P. D. et al. Ultramicroscopy 151 (2015), p. 160-167.

[10] Jiang Y., Chen Z., Muller D. A. et al. Nature 559 (2018), p. 343-349. 